\title{
Influência de compostos de boro nas propriedades físicas da madeira de Eucalyptus sp.
}

\author{
Influence of boron compounds on the physical \\ properties of Eucalyptus sp. wood
}

\author{
Edy Eime Pereira Baraúna1, Amanda Mota Nunes², Jordão Cabral Moulin³, \\ Thiago Campos Monteiro', Marina Donária Chaves Arantes ${ }^{4}$ e Juarez Benigno Paes ${ }^{5}$
}

\begin{abstract}
Resumo
Para impedir a degradação da madeira por coleópteros e cupins pode-se fazer o uso de diversas substâncias químicas, entre as quais se destacam os compostos de boro. Seus efeitos nas propriedades físicas da madeira são pouco conhecidos.Desse modo, o objetivo desta pesquisa foi identificar a influência dos compostos de boro na densidade básica e retração da madeira de Eucalyptus sp. e a eficiência do tratamento preservativo. Foram usados 160 corpos de prova com dimensões de $2 \times 2 \times 5 \mathrm{~cm}$, provenientes de resíduos madeireiros, em que 80 amostras foram tratadas com ácido bórico e outros 80 não receberam tratamento químico. Após o tratamento de impregnação a vácuo, realizou-se o teste qualitativo para avaliar a penetração do boro na madeira tratada, bem como os ensaios de densidade e retração nas amostras com e sem tratamento químico. Os resultados indicaram que a maior penetração na madeira tratada com compostos de boro foi classificada como parcial irregular e, que o tratamento preservativo aumentou significativamente a densidade básica da madeira tratada, em relação à não tratada. Os valores médios de retração total na madeira tratada foram inferiores em 17,5\%, 14,8\% e 15,5\%, para retração tangencial, radial e volumétrica, respectivamente, em relação à madeira não tratada. Desse modo, observou-se que o boro afetou significativamente as propriedades físicas da madeira analisada.
\end{abstract}

Palavras-chave: Estabilidade dimensional; ácido bórico; densidade básica; tratamento preservativo.

\begin{abstract}
Order to prevent the wood degradation with coleopteran and termites can make use of various chemical preservatives, which are highlighted the boron compounds. Their effects on physical properties of wood are little known.Thus, the aim of this research was to identify the influence of boron compounds in specific gravity and skrinkage of Eucalyptus sp. and the effectiveness of the preservative treatment. A total of 160 specimens were used test pieces with dimensions of $2 \times 2 \times 5 \mathrm{~cm}$ from wood residues, where 80 samples were treated with boric acid and other 80 received no treated. After vacuum impregnation treatment, the qualitative test was performed to evaluate the penetration of boronin treated wood, the density and skrinkage assays in samples with or without preservative. The results indicated that greater penetration of the wood treated with boron compounds was classified as partially irregular level, and the preservative treatment significantly increased the specific gravity in treated samples as compared to the untreated. Mean values of total skrinkage in the treated wood were lower by $17.5 \%, 14.8 \%$ and $15.5 \%$, tangential, radial and volumetric skrinkages, respectively, as compared to untreated wood. Thus, it was observed that boron significantly affect the physical properties of evaluated wood.
\end{abstract}

Keywords: Dimensional stability; boric acid; specific gravity; preservative treatment.

1Professor Adjunto do Departamento de Engenharia Florestal. UFMG - Universidade Federal de Minas Gerais / Instituto de Ciências Agrárias. Avenida Universitária Universitário - 39404547 - Montes Claros, MG, Brasil. E-mail: ebarauna@ufmg.br; tcmforest@yahoo.com

2Engenheira Florestal, Mestre em Ciências Florestais. UFES - Universidade Federal do Espírito Santo / Av. Gov. Lindemberg, 316 - 29550-000 - Jerônimo Monteiro, ES, Brasil. E-mail: amanda.mota@live.com

32Doutorando em Ciência e Tecnologia da Madeira. UFLA - Universidade Federal de Lavras. Av. Doutor Sylvio Menicucci, 1001 - 37.200-000 - Lavras, MG, Brasil. E-mail: jordao cm@hotmail.com

${ }^{4}$ Professor Adjunto no Departamento de Ciências Florestais. UFES - Universidade Federal do Espírito Santo / Av. Gov. Lindemberg, 316 - 29550-000 - Jerônimo Monteiro, ES, Brasil. E-mail: mdonariac@hotmail.com

${ }^{5}$ Professor Titular do Departamento de Ciências Florestais. UFES - Universidade Federal do Espírito Santo / Av. Gov. Lindemberg, 316 - 29550-000 - Jerônimo Monteiro, ES, Brasil. E-mail: jbp2@uol.com.br

Sci. For., Piracicaba, v. 45, n. 113, p. 197-204, mar. 2017 DOI: dx.doi.org/10.18671/scifor.v45n113.20 


\section{INTRODUÇÃO}

A madeira é um material muito utilizado pelo homem desde os primórdios da sociedade humana até os dias atuais nos mais diferentes segmentos, como na construção de abrigos, fabricação de armas, utensílios, ferramentas, esquadrias e na construção civil (MIOTTO, 2002). Entretanto, quando em serviço, a mesma é susceptível ao ataque de organismos xilófagos, os quais causam prejuízos econômicos ao homem (BARILLARI, 2002). E, para impedir tal degradação, atualmente faz-se uso de diversos produtos químicos, entre os quais se destacam os compostos de boro.

Os compostos do boro são úteis na preservação da madeira por causa de suas características de ambientalmente seguro ao homem, baixo custo e efetividade contra os agentes xilófagos (bactérias, fungos, coleópteros e cupins) além da conhecida propriedade de resistência ao fogo (YALINKILIC et al., 1999). Dentre os vários tipos de substâncias preservativas citadas por Santini (1988) e Lepage (1986), destaca-se a mistura de bórax com ácido bórico.

Madeiras contendo $0,2 \%$ de ácido bórico equivalente (BAE) estão protegidas contra diversos organismos xilófagos (OLIVEIRA, 1990). Entretanto, tal taxa de retenção do ácido bórico pode ser influenciada quando se utiliza madeira de baixa permeabilidade, cerne refratário e de alta densidade. Um processo indicado para o tratamento de madeiras que apresentem cerne refratário e baixa permeabilidade é o método a vácuo (VEENIN; VEENIN, 2001). Com este processo podem-se obter adequadas penetrações do produto na madeira, além de ser considerado um método econômico, principalmente quando se compara os custos de investimentos com os resultados obtidos.

A escolha do método ou processo de tratamento a ser utilizado são fatores que irão afetar a eficiência do procedimento, porém há um terceiro fator, de fundamental importância, que são as características da madeira (AMARAL, 2012). Os compostos de boro podem alterar as propriedades mecânicas, físicas, biológicas e resistência ao fogo da madeira sólida e painéis reconstituídos (BAYSAL et al., 2007; KARTAL et al., 2007; USTA et al., (2009)

De acordo com Dos Santos (2010), as propriedades físicas mais estudadas, por serem as mais importantes e por pré-estabelecerem um conhecimento prévio da madeira são a retratibilidade e a densidade. No entanto, no Brasil, consta-se uma quantidade bastante reduzida de pesquisas direcionadas à influência do processo preservativo, usando compostos de boro, em tais propriedades da madeira.

A densidade da madeira é uma quantificação direta do material lenhoso por unidade de volume, e está relacionada à maioria das propriedades e características tecnológicas fundamentais para a produção e utilização de produtos florestais. Trata-se de um dos parâmetros mais importantes entre as diversas propriedades físicas da madeira, pois afeta todas as demais (AMARAL, 2012). Em geral, a densidade básica da madeira de Eucalyptus spp. varia entre 0,5 a 0,8 $\mathrm{g} \mathrm{cm}^{-3}$ (SEVERO, 2000).

A retração e a expansão higroscópica da madeira são dois dos mais importantes problemas práticos que ocorrem durante a sua utilização, como consequência da mudança do teor de umidade. Todo material higroscópico, como a madeira e vários outros materiais lignocelulósicos, apresenta retração quando o seu teor de umidade do ponto de saturação das fibras (PSF) é reduzido. A magnitude das variações dimensionais depende de inúmeros fatores, como o teor de umidade, a direção estrutural (radial, tangencial ou longitudinal), a posição dentro da árvore, a densidade da madeira, a temperatura e o grau de estresse de secagem causada pelo gradiente de umidade (OLIVEIRA; SILVA, 2003).

Desse modo, pesquisas considerando a importância do estudo das propriedades físicas (densidade e retratibilidade) de madeiras impregnadas com boro são justificáveis. Sendo assim, o objetivo deste estudo foi avaliar a influência de compostos de boro na densidade e retração da madeira de Eucalyptus spp. e a qualidade do tratamento preservativo pelo método a vácuo.

\section{MATERIAL E MÉTODOS}

\section{Obtenção do material e impregnação dos corpos de prova}

Os corpos de prova utilizados neste estudo foram obtidos a partir de costaneiras geradas do processamento mecânico da madeira de Eucalyptus spp., por indústria madeireira localizada no município de Gurupi - TO. Os corpos de prova usados para impregnação com boro (tratado) e não 
tratados, totalizaram 160 amostras, com dimensões de $2 \times 2 \times 5 \mathrm{~cm}$.As amostras em seguida foram acondicionadas em sala climatizada, mantida na temperatura de $20 \pm 2^{\circ} \mathrm{C}$ e umidade relativa de 65 $\pm 5 \%$, até massa constante.

Após a climatização dos corpos de prova, os mesmos foram levados para o Laboratório de Tecnologia de Produtos Florestais - LTPF do Campus Universitário de Gurupi, Universidade Federal do Tocantins - UFT, onde se confeccionou um aparato para impregnação a vácuo, a partir de adaptações da American Wood Protection Association Standard - AWPA - E10 (AWPA, 2008).

A solução preservativa usada na impregnação dos corpos de prova consistiu de uma mistura de 4\% de ácido bórico e bórax, ou seja, 40g de cada composto para $2000 \mathrm{~mL}$ de água destilada, preparada segundo metodologia descrita por Veenin e Veenin (2001). Após o preparo da solução, as amostras foram colocadas em um béquer para que fossem submetidas a um vácuo inicial de 680 mmHg até estabilização, para posteriormente ser liberada a entrada da solução preservativa.

O tempo de impregnação consistiu em dois vácuos de 30 minutos, intercalados por um intervalo de 40 minutos cada, e a quantidade de solução usada na impregnação de cada lote de amostras foi de $800 \mathrm{~mL}$, quantidade requerida para que houvesse a imersão uniforme de todos os corpos de prova. O processo de impregnação foi realizado segundo a AWPA - E10 (AWPA, 2008).

Após a impregnação, a fim de que houvesse uma melhor fixação da solução preservativa na madeira, os corpos de prova foram acondicionados em filme de polietileno e deixados em repouso por aproximadamente 14 dias. Transcorrida a etapa de fixação, foi removido o filme para a secagem ao ar livre até massa constante, para, em seguida, realizar a secagem das amostras em estufa por um período de sete dias, a uma temperatura inicial de $40^{\circ} \mathrm{C}$, a qual foi gradativamente aumentada até atingir a temperatura final de $103 \pm 2{ }^{\circ} \mathrm{C}$. Esse processo de secagem foi adotado de maneira a evitar a presença de colapso e rachaduras nas amostras.

\section{Determinação da densidade básica e retração da madeira}

Com o uso de paquímetro digital, com precisão de 0,01 mm, e balança analítica de 0,0001g de precisão, foram obtidas dos corpos de prova tratados e não tratados as dimensões e massas. As medidas foram tomadas antes da realização de qualquer procedimento, e nos momentos em que as amostras se encontravam com a umidade acima do PSF, em umidade de equilíbrio e absolutamente secas em estufa. Desse modo, e de posse de todas as medidas de cada amostra (tratamento e controle) estabeleceram-se os dados para caracterização física das amostras analisadas e comparadas no estudo.

A densidade básica foi determinada conforme a Norma Brasileira Regulamentadora - NBR 11941, Associação Brasileira de Normas Técnicas - ABNT (2003). Considerou-se o volume verde das amostras tratadas, aquele tomado na condição de pós impregnação, e o das amostras controle, o saturado em água.

A análise da retração da madeira foi realizada como índice de estabilidade dimensional, de acordo com a NBR 7190 (ABNT, 1997), em que foram obtidas as retrações tangencial, radial e volumétrica, como também o coeficiente de anisotropia. O ensaio foi realizado a partir de sucessivas medições nos eixos longitudinal, radial e tangencial de cada corpo de prova, tratado e não tratado. Para isso, consideraram-se as dimensões tomadas quando em umidade acima do PSF e a $0 \%$ de umidade.

\section{Teste qualitativo da penetração do boro na madeira}

A fim de se observar a qualidade do tratamento preservativo adotado, realizou-se o teste qualitativo com base na penetração do boro na madeira tratada. Para tanto, os corpos de prova foram seccionados na metade do seu comprimento, ficando com dimensões de 2 × 2 × $2 \mathrm{~cm}$, de modo a observar a impregnação na parte transversal central dos mesmos. Neste teste, utilizou-se de apenas uma das amostras obtida por corpo de prova impregnado, totalizando 80 repetições.

Neste método empregaram-se soluções de curcumina e de ácido salicílico para observar a penetração do boro na madeira. Para a primeira solução, dissolveu-se 0,12 g de curcumina em etanol morno, temperatura de 40 a $42^{\circ} \mathrm{C}$, esfriado e diluído para $100 \mathrm{~mL}$ com etanol, e para a segunda, dissolveram-se $6 \mathrm{~g}$ de ácido salicílico em $20 \mathrm{~mL}$ de ácido clorídrico concentrado, completando-se para 100 mL com etanol (WILLIAMS, 1968a; BARAÚNA et al., 2011). 
A aplicação das soluções nos corpos de prova previamente impregnados ocorreu em dois estágios. Aplicação da solução de curcumina nas amostras por pulverização, na face transversal exposta, de forma a não deixar escorrer o reagente pela amostra. Após a secagem da solução de curcumina, borrifou-se a solução de ácido salicílico. Após a mudança de cor, causada pela reação do ácido salicílico com a curcumina produzindo o complexo vermelho-resorcianina, foi avaliada a coloração final obtida. De acordo com Smith e Williams (1969), quando ocorre alteração da cor da madeira para vermelho indica a presença de mais de $0,2 \%$ de ácido bórico equivalente na substância preservativa impregnada na amostra.

A análise da penetração do boro foi realizada com base na descrição de Lima (2012) acerca das possibilidades de penetração do produto químico na madeira, com base nos padrões propostos por Sales-Campos et al. (2003) (Figura 1), que estabelece as seguintes classes de penetração: (1) Penetração total, quando toda extensão do alburno é penetrada pelo produto; (2) Penetração parcial periférica, quando toda a periferia da madeira é penetrada, embora não atinja profundidade total na madeira; (3) Penetração parcial e irregular, quando uma parte da periferia da peça é penetrada mas, uma das faces periférica fica sem penetração de preservativo; (4) Penetração vascular, quando o produto químico penetra apenas pelos vasos da madeira, sem atingir outras partes do lenho; e (5) penetração nula, quando não há nenhuma penetração do produto no lenho.

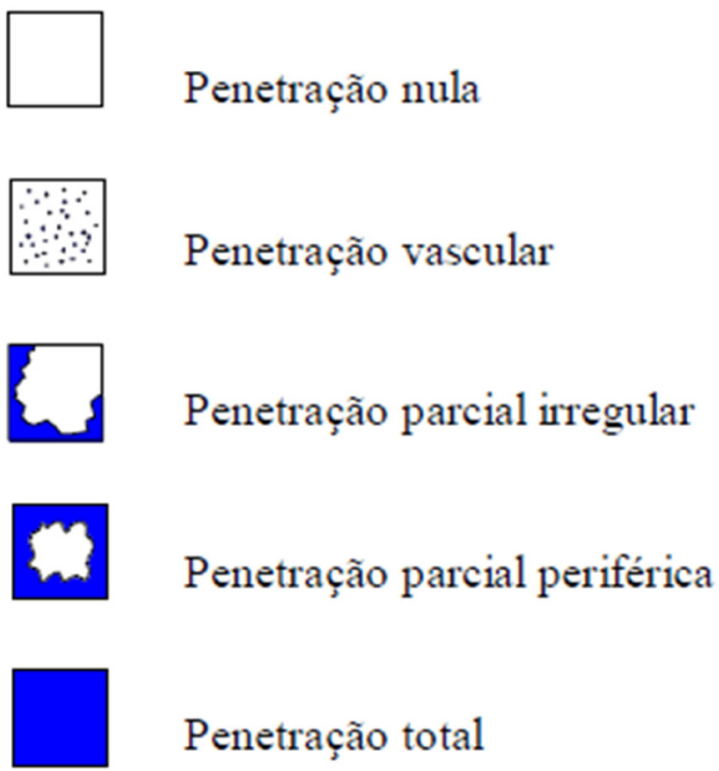

Figura 1. Padrões mais comuns de penetração do teste de tratabilidade de madeira. Fonte: Sales-Campos et al. (2003).

Figure 1. Common Patterns of penetration of wood treatability test. Source: Sales-Campos et al. (2003).

\section{Análise estatística dos dados}

Para a comparação dos dados e a observação da influência do tratamento preservativo nas propriedades físicas das madeiras tratada e não tratada, procedeu-se a análise estatística descritiva dos dados e a de variância, ao empregar um delineamento inteiramente casualizado.

Como se tratou de apenas duas situações (madeira tratada e na tratada), não foi necessário à aplicação de teste para a discriminação de médias, pois neste caso, o teste $\mathrm{F}$, já é o suficiente para tal finalidade. O mesmo foi aplicado a 95\% de probabilidade, utilizando o software estatístico R, versão 2.11.0 (R DEVELOPDMENT CORE TEAM, 2008).

\section{RESULTADOS E DISCUSSÃO}

\section{Densidade básica e retração da madeira}

Verifica-se que a densidade básica da madeira tratada foi superior a não tratada (Tabela 1). Tal situação, possivelmente, pode ser explicada pela presença dos compostos de boro na madeira tratada, os quais estão influenciando na sua massa quando seca, aumentando assim a sua densidade em relação à madeira não tratada. 
Tabela 1. Densidade básica, retração e anisotropia obtidas para a madeira de Eucalyptus sp.

Table 1. Basic density, shrinkage and anisotropy obtained for the Eucalyptus sp.

\begin{tabular}{lccccc}
\hline \multirow{2}{*}{ Tratamento } & Densidade & \multicolumn{3}{c}{ Retração (\%) } & Coeficiente de \\
\cline { 3 - 5 } & Básica $\left(\mathbf{g} / \mathbf{c m}^{3}\right)$ & Tangencial & Radial & Volumétrica & anisotropia \\
\multirow{2}{*}{ Tratada } & $0,514 \mathrm{a}$ & $5,86 \mathrm{~b}$ & $4,82 \mathrm{~b}$ & $10,61 \mathrm{~b}$ & $1,23 \mathrm{a}$ \\
& {$[10,3]$} & {$[28,2]$} & {$[22,1]$} & {$[21,7]$} & {$[23,6]$} \\
\hline \multirow{2}{*}{ Não Tratada } & $0,497 \mathrm{~b}$ & $7,10 \mathrm{a}$ & $5,66 \mathrm{a}$ & $12,56 \mathrm{a}$ & $1,28 \mathrm{a}$ \\
& {$[9,7]$} & {$[19,5]$} & {$[20,7]$} & {$[16,8]$} & {$[22,5]$} \\
\hline
\end{tabular}

Médias seguidas da mesma letra não diferem significativamente entre si, pelo teste $F(p>0,05)$. Valores entre colchetes são os coeficiente de variação.

Kollmann e Cotê Junior (1968) afirmaram que, quanto mais alta a densidade da madeira, maior a sua retração ou expansão, principalmente para o caso do gênero Eucalyptus. Porém, como descrito por Oliveira (2003), há controvérsia em tal afirmação. No caso deste trabalho, o aumento da densidade deve-se à presença do boro, possivelmente, nos espaços como vasos, células parenquimáticas e espaços intercelulares. Assim, além deste aumento da densidade não ter resultado no aumento da retração da madeira tratada, o boro pode ter dificultado a retração desta madeira durante a secagem.

Outro fato a ser discorrido sobre os valores de retração encontrados neste estudo, tanto para a madeira tratada quanto para a não tratada, é de que os mesmos são inferiores aos encontrados por Muller et al. (2014), os quais correspondem, nesta ordem, a 13,87; 5,91 e 18,52\% para as contrações nas direções tangencial, radial e volumétrica, para a madeira de Eucalyptus benthamii.

Quanto aos valores médios de coeficiente de anisotropia (Tabela 1), nota-se que os mesmos não apresentam diferenças significativas. No entanto, ao se levar em consideração inúmeras afirmações acerca da estabilidade da madeira de Eucalyptus spp., observa-se que os valores de coeficiente de anisotropia obtidos neste estudo são consideravelmente baixos, comparados aos valores encontrados por Gonçalves et al. (2009) e por Silva et al. (2006), para madeiras de um híbrido clonal de Eucalyptus urophylla $\mathrm{x}$ Eucalyptus grandis de duas idades e, Eucalyptus grandis com quatro diferentes idades, respectivamente.

Oliveira (1997) afirmou que de forma geral, há uma tendência em todas as espécies de eucalipto, de que a madeira apresente valores mais baixos para o fator anisotrópico próximo ao alburno, o que indicaria maior estabilidade dimensional. Pela razão do material usado neste estudo ser proveniente de costaneiras, onde há a possibilidade de conter apenas alburno, tem-se a explicação dos valores de anisotropia obtidos, os quais podem estar sendo influenciados pela presença deste tipo de lenho nas amostras.

Um coeficiente de anisotropia de retração igual a 1 representa uma alteração igual de dimensões nos sentidos radial e tangencial, situação considerada ideal e que não provocaria formação de tensões internas durante o crescimento da planta (DURLO; MARCHIORI, 1992).

Nota-se ainda (Tabela 1), que o coeficiente de variação na madeira tratada é superior a não tratada. Tal fato expressa que o lote de amostras apresenta uma maior heterogeneidade em relação ao outro. Considerando que os corpos de prova usados para comparação são provenientes de uma mesma peça, pressupõe-se que a solução preservativa possa estar exercendo influência sobre características anatômicas que se correlacionam com as propriedades analisadas ou, ainda, esteja ocupando diferentes regiões dentro da madeira, como os elementos vasculares e células parenquimáticas, aumentando a densidade sem apresentar relação de proporcionalidade desta, com as contrações da madeira.

Entretanto, para tal afirmação, é necessário um estudo mais aprimorado da anatomia da madeira quando tratada com compostos de boro, bem como o efeito da composição deste mineral dentro da madeira.

\section{Penetração do boro nas amostras tratadas}

Observa-se que, de modo geral, o tratamento preservativo foi eficiente na impregnação de boro da madeira de Eucalyptus spp. A cor vermelha indica a presença de 0,30\%, o vermelho-amarelo indica $0,25 \%$ e a não mudança de cor equivale a um valor abaixo de $0,15 \%$ de boro ou a sua ausência (WILLIAMS, 1968b; WILLERDING; VIANEZ, 1998). A partir da classificação visual (SALES-CAMPOS et al., 2003) das amostras, notou-se que 30\% apresentaram penetração total, com colorações 
avermelhadas, porém mais claras em relação às demais. Já 43\% das amostras tiveram penetrações parciais irregulares.

Borges (2008), em estudo realizado com a madeira de Corymbia citriodora com 18 anos de idade, tratada em autoclave pelo processo de Bethell com CCA, observou a mesma tendência encontrada no trabalho realizado por Vivian et al. (2012) para a madeira de Eucalyptus cloeziana, em que o alburno foi totalmente tratado, entretanto, na parte da peça com cerne não houve penetração do produto, de forma que a mesma enquadrou-se como parcial irregular.

Desse modo, em virtude do tratamento da madeira de Eucalyptus spp. ter sido realizado em nível experimental, e de grande parte das amostras terem apresentado penetração do tipo periférica e manifestado coloração avermelhada, considera-se que a penetração obtida foi satisfatória. De acordo com Willians (1968a) e Willerding e Vianez (1998), as amostras, supostamente, contariam com a presença de $0,3 \%$ de boro em sua constituição depois de impregnadas. Tais valores indicam que há uma garantia de proteção mais eficaz da madeira, contra o ataque de coleópteros e cupins (FREEMAN et al., 2009)

\section{CONCLUSÕES}

Conclui-se que os compostos de boro influenciaram positivamente a densidade básica da madeira tratada.

Embora o tratamento preservativo tenha aumentado a densidade básica da madeira tratada, esta apresentou valores médios de retração, nos diferentes eixos, menores que a madeira não tratada.

O tratamento preservativo da madeira com compostos de boro a partir do método a vácuo foi eficiente para o tratamento de madeira de Eucalyptus spp.

A maior penetração apresentada pela madeira tratada com compostos de boro foi em nível parcial irregular.

\section{REFERÊNCIAS BIBLIOGRÁFICAS}

AMARAL, L. S. Penetração e retenção do preservante em Eucalyptus com diferentes diâmetros. 2012. 81 p. Dissertação (Mestrado em Ciências e Tecnologia da Madeira) - Universidade Federal de Lavras, Lavras.

ABNT - ASSOCIAÇÃO BRASILEIRA DE NORMAS TÉCNICAS. NBR 11941: Madeira - Determinação da densidade básica. Rio de Janeiro, 2003. 6p.

ABNT - ASSOCIAÇÃO BRASILEIRA DE NORMAS TÉCNICAS. NBR 7190: Projeto de estruturas de madeira. Anexo B - Determinação das propriedades das madeiras para projetos de estruturas. Rio de Janeiro, 1997. $107 \mathrm{p}$.

AWPA - AMERICAN WOOD PROTECTION ASSOCIATION STANDARD. AWPA - E10: Testing wood preservatives by laboratory soil-block cultures. Birmingham, 2008. $10 \mathrm{p}$.

BARAÚNA, E. E. P.; VIEIRA, R. S.; MONTEIRO, T. C.; ARANTES, M. D. C.; DE CARVALHO, D. M.; LIMA, M. T. Tratamento térmico de madeira serrada do angelim pedra (Dinizia excelsa Ducke) com boro. Journal of Biotechnology and Biodiversity, v.2, n.1, p. 30-36, 2011.

BARILLARI, C. T. Durabilidade da madeira do gênero Pinus tratada com preservantes: avaliação em campo de apodrecimento. 2002. 68 p. Dissertação (Mestrado em Recursos Florestais) - Escola Superior de Agricultura Luiz de Queiroz, Piracicaba.

BAYSAL, E.; ALTINOK, M.; OZAKI, S. K.; TOKER, H. Fire resistance of Douglas fir (Pseudotsuga menziesii)treated with borates and natural extractives. Bioresource Technology, Essex, v. 98, n. 5, p. 1101-1105, 2007.

BORGES, C.C. Potencialidade do uso de cruzetas de madeira tratada. 2008. 107 p. Dissertação (Mestrado em Ciências Florestais) - Universidade Federal do Paraná, Curitiba, 2008. 
SANTOS, H. S. Padronização de ensaios para identificação de preservantes em postes de madeira e solos de áreas controladas. 2010. 109 p. Dissertação (Mestrado em Engenharia de Materiais) - Pontifícia Universidade Católica do Rio Grande do Sul, Porto Alegre.

DURLO, M. A.; MARCHIORI, J. N. C. Tecnologia da madeira: retratibilidade. Santa Maria: CEPEF/FATEC, 1992, v. 33.

FREEMAN, M.H.; MCINTYRE, C.R.; JACKSON, D. A critical and comprehensive review of boron in wood preservation. AWPA, v.105, p. 279-294, 2009.

GONÇALVES, F. G.; OLIVEIRA, J. T. S.; LUCIA, R. M. D.; NAPPO, M. E.; SARTÓRIO, R. C. Densidade básica e variação dimensional de um híbrido clonal de Eucalyptus urophylla x Eucalyptus grandis. Revista Árvore, Viçosa, v. 33, n. 2, p.277-288, 2009.

KARTAL, S. N.; AYRILMIS, N.; IMAMURA, Y. Decay and termite resistance of plywood treated with various fire retardants. Build and Environment,v. 42, n. 3, p. 1207-1211, 2007.

KOLLMANN, F. F. P.; CÔTÉ JUNIOR, W. A. Principles of wood science and technology. New York: SpringerVerlag, 1968. 592 p. v.1.

LEPAGE, E. S. (Coord.). Manual de preservação de madeira. São Paulo: Instituto de Pesquisa Tecnológicas, 1986. $708 \mathrm{p}$.

LIMA, F. C. C. Avaliação de nove espécies de Eucalyptusspp. em tratamento preservativo industrial. 2012. 82 p. Dissertação (Mestrado em Ciência Florestal) - Universidade Estadual Paulista "Júlio de Mesquita Filho", Botucatu, 2012.

MIOTTO, J. L. Evolução das esquadrias de madeira no Brasil. Ciências Exatas e Tecnologia, Londrina, v. 1, n. 1, p. $55-62,2002$.

MULLER, B. V.; ROCHA, M. P.; CUNHA, A. B.; KLITZKE, R. J.; NICOLETTI, M. F. NICOLETTI, M. F. avaliação das principais propriedades físicas e mecânicas da madeira de Eucalyptus benthamii Maiden et Cambage. Floresta e Ambiente, Seropédica, v. 21, n. 4, p. 535-542, 2014.

OLIVEIRA, J. T. S. Caracterização da madeira de eucalipto para a construção civil. 1997. 429 p. Tese (Doutorado em Engenharia Civil) - Escola Politécnica da Universidade de São Paulo, São Paulo.

OLIVEIRA, V. S. Mechanisms of diffusion of boron through wood. 1990. 136 p. Tese (Philosophia e Doctor in Agricultural and Forest Sciences) - University of Wales, Bangor.

OLIVEIRA, J. T. S.; SILVA, J. C. Variação radial da retratibilidade e densidade básica da madeira de Eucalyptus saligna Sm. Revista Árvore, v. 27, n.3, p.381-385, 2003.

R DEVELOPMENT CORE TEAM. R: a language and environment for statistical computing. Vienna: R Foundation for Statistical Computing, 2014.

SALES-CAMPOS, C.: VIANEZ, B, F:: MENDONÇA, M. S. Estudo da variabilidade da retenção do preservante CCA tipo A na madeira de Brosimum rubescens Taub. Moraceae - (pau-rainha) uma espécie madeireira da região Amazônica. Revista Árvore, Viçosa, v.27, n.6, p.845-853, 2003.

SANTINI, E. J. Biodeterioração e preservação da madeira. Santa Maria: UFSM, 1988. 120 p.

SEVERO, E. T. D. Qualidade da madeira serrada de Eucalyptus dunnii. Ciência Florestal, Santa Maria, v.10, n.1, p.109-124, 2000. 
Baraúna et al. - Influência de compostos de boro nas propriedades físicas da madeira de Eucalyptus sp.

SILVA, J. C.: OLIVEIRA, J. T. S.: XAVIER, B. A.: CASTRO, V. S. Variação da retratibilidade da madeira de Eucalyptus grandis Hill ex. Maiden, em função da idade e da posição radial no tronco. Revista Árvore, Viçosa, v.30 n.5, p.803-810, 2006.

SMITH, D. N. R.; WILLIAMS, A. I. Wood preservation by the boron diffusion process-the effect of moisture content on diffusion time. Glasgow: Building Research Establishment, 1969.11 p. (Timberlab Paper 5).

USTA, M.; USTAOMER, D.; KARTAL, N.; HAGIO, L. Termite resistance of MDF panels treated with various boron compounds. International Journal of Molecular Sciences, v. 10, n. 6, p. 2789-2797, 2009.

VEENIN, A.; VEENIM, T. A Laboratory study on effect of coating materials on leaching of copper from CCA treated wood. Stockholm: The International Research Group on Wood Protection, 2001. 13 p.

VIVIAN, M. A.: SANTINI, E. J.: MODES, K. S.: MORAIS, W. W. C. Qualidade do tratamento preservativo em autoclave para a madeira de Eucalyptus grandis e Eucalyptus cloeziana. Scientia Forestalis, Piracicaba, v.40, n. 96, p. 445-453, 2012.

WILLERDING, A. L.; VIANEZ, B. F. Utilização de bórax por difusão no tratamento depreservação de lâminas de sumaúma (Ceiba pentantra (L.) Gaertn.). Revista Árvore, Viçosa, v. 27, n. 3, p. 321-326, 2003.

WILLIAMS, A. I. Determination of disodium octaborate in thin sections of diffusion treated wood. Forest Products Journal, Madison n. 93, p. 111-112, 1968a.

WILLIAMS, A. I. The extraction and determination of disodium octaborate in Sitka spruce. Analyst, n.93, p. 111-115, 1968b.

YALINKILIC, M. K.; IMAMURA, Y.; TAKAHASHI, M.; YALINKILIC, A. C. In situ polymerization of vinyl monomers during compressive deformation of wood treated with boric acid to delay boron leaching. Forest Products Journal, Madison, v. 42, n. 2, p. 43-51, 1999.

Recebido em 21/01/2016

Aceito para publicação em 31/01/2016 